\section{A nervous disposition: the angiometabolic switch}

New data, published in Science, show that adrenergic nerves can activate an angiometabolic switch in prostate cancer. Activation of this switch promotes tumour growth via altered endothelial cell metabolism; thus, crosstalk between nerves and endothelial cells, affecting metabolism, could be a target for anticancer therapy.

In wild-type mice, orthotopically implanted prostate cancer xenografts grew exponentially after day 18; however in mice deficient in Adrb2 and Adrb3, no substantial tumour growth was observed after this time point. These knockout mice did initially develop tumours, and no difference in tumour size, vascular permeability, or hypoxic area was seen in wild-type and knockout mice before day 18 . Further analysis revealed that tumours from knockout mice had a lower density of tumour vessels than their wild-type counterparts, caused by considerable alterations in vascular patterns (such as reduced length and branching) in the prostate. Orthotopically transplanting cell-free collagen I (an angiogenic superpolymer) matrix into the prostate capsule resulted in reduced vessel migration, density, and branching in chemically sympathectomized mice.

Analysis of high-grade prostatic intraepithelial neoplasia (PIN) lesions that form at 8 weeks in a $\mathrm{Hi}-\mathrm{Myc}$ spontaneous autochthonous prostate cancer mouse model showed that nerve and blood vessel density and proximity were increased compared with low-grade PIN lesions that are present at 4 weeks. Furthermore, levels of noradrenaline (a $\beta$-adrenergic neurotransmitter) were significantly increased in $\mathrm{Hi}$-Myc mice compared with wild-type mice.

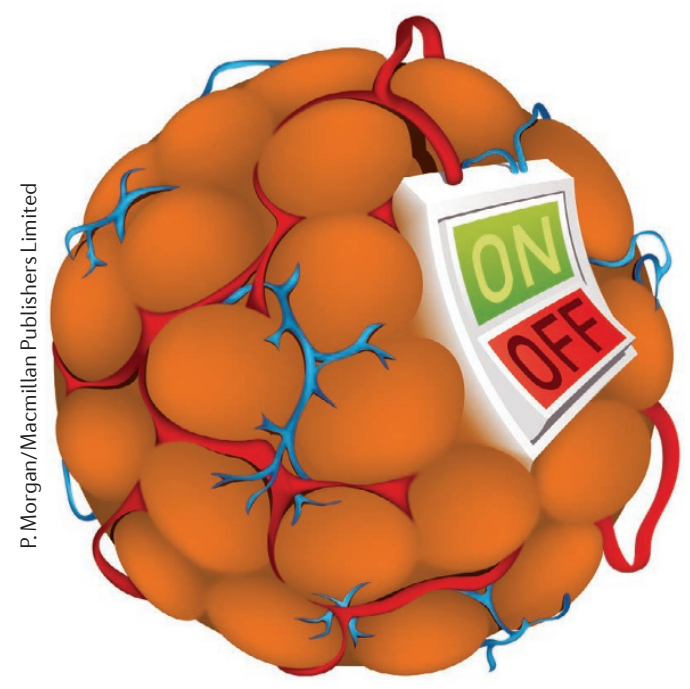

Molecular investigation showed that expression of Adrb2 was highest in endothelial cells among the stromal cell population. Conditional deletion of Adrb2 in the endothelial cells of Hi-Myc mice during low-grade PIN development inhibited progression to high-grade PIN, which was sustained for $>12$ months. A reduction in proliferative endothelial cells to levels similar to those observed in low-grade PIN was observed in Hi-Myc mice with conditional endothelial cell Adrb2 knock out. Adrb2 deletion during the low-grade PIN stage resulted in reduced vessel density, migration, and branching at 8 weeks.

Gene set enrichment analysis revealed an increase in mitochondrial cytochrome $c$ activity and expression of Bckdha and Coa6. Endothelial mitochondrial membrane potential was increased and the NADH quotient reduced in endothelial cells from Adrb2-deficient Hi-Myc mice, indicating enhanced oxidative phosphorylation. Oxidative phosphorylation was also increased in xenografts from Adrb2-knockout Hi-Myc and denervated wild-type mice. In vitro, mouse prostate epithelial cells deficient in Adrb2 had increased glucose Furthermore, no changes in ATP levels were observed in Adrb2-deficient cells when glucose was replaced with galactose in the culture medium. The migratory capacity of Adrb2deficient cells was reduced and microtubule orientation was altered.

Overexpression of Coa6 in prostate epithelial cells increased oxidative phosphorylation and inhibited cell migration and proliferation. In vivo, orthotopic cotransplantation of Coa6-GFP endothelial cells with PC-3 cells resulted in reduced tip cell formation and angiogenesis.

These data show that adrenergic nerve activity affects endothelial cell metabolism, influencing angiogenesis and prostate cancer progression. Inhibiting $\beta$-adrenergic signalling could be a therapeutic strategy for treating this disease. To this end, Paul Frenette, corresponding author, told Nature Reviews Urology "We have developed a pilot study to assess the effect of $\beta$-blockers in patients just diagnosed with prostate cancer who will undergo prostatectomy." He concluded "This study pilot could provide proof-of-principle data in humans for larger prospective trials."

Louise Stone uptake, and ATP, Coa6, and citrate levels. (2017) adrenergic

nerve activity

affects

endothelial cell

metabolism, influencing angiogenesis 